\title{
GPS Operations in High Earth Orbit: Recent Experiences and Future Opportunities
}

\author{
Benjamin W. Ashman ${ }^{1}$ \\ Goddard Space Flight Center, Greenbelt, MD, 20771, USA \\ Frank H. Bauer ${ }^{2}$ \\ FBauer Aerospace Consulting Services, Silver Spring, MD, 20905, USA \\ Joel J. K. Parker ${ }^{3}$ and Jennifer E. Donaldson ${ }^{4}$ \\ Goddard Space Flight Center, Greenbelt, MD, 20771, USA
}

\begin{abstract}
Over the past two decades, spacecraft in Low Earth Orbit (LEO) have significantly benefited from real-time reception of navigation and timing signals from the Global Positioning System (GPS). By employing GPS receivers that are specially developed to support reception in space, LEO spacecraft now realize significantly reduced recovery time after trajectory maneuvers, improved operations cadence, increased satellite autonomy, and more precise, real-time navigation and timing performance. These benefits are now being extended beyond LEO: despite extremely weak signal reception and less favorable geometry, a number of upcoming High Earth Orbit (HEO) missions are also poised to benefit from improved navigation, timing, and onboard autonomy thanks to GPS. This paper will describe the results of two recent missions (MMS and GOES-16), provide an understanding of the benefits and limitations of GPS beyond LEO, and outline future missions and opportunities where this capability would result in significant and enabling benefits.
\end{abstract}

\section{Introduction}

Space-borne Positioning, Navigation and Timing (PNT) researchers have been aggressively expanding the use of the Global Positioning System (GPS) on space vehicles. Starting with nascent space flight experiments in LowEarth Orbit (LEO) in the 1980s and 1990s, space-borne GPS is now commonplace in this orbit regime [1]. PNT researchers are now expanding GPS use into - and beyond - the Space Service Volume (SSV), the volume surrounding the Earth at altitudes above 3,000 km that supports high-altitude, real-time GPS navigation and timing. Expansion into the SSV has spawned exciting new operational missions through radically improved navigation and timing performance, quick trajectory maneuver recovery, and improved space vehicle autonomy. These operational missions have demonstrated outstanding PNT performance characteristics, much better than what was envisioned less than a decade ago.

\section{A. GPS Space User Operational Environment: The Terrestrial and Space Service Volumes}

GPS employs a constellation of at least 24 satellites in Medium Earth Orbit (MEO), transmitting one-way radio signals that are used to calculate three-dimensional position, velocity, and time, primarily for Earth and near-Earth users. Traditionally, at least four GPS satellites are needed to be within line-of-sight at any given time to form a point solution. But innovations developed to support space users at high altitudes — where GPS signals are sparse — enable GPS solutions with as few as one signal in view.

Requirements for GPS performance in space have been allocated to two service volumes: the Terrestrial Service Volume (TSV), which includes all terrestrial users and Low Earth Orbit (LEO) space users and extends to an altitude of $3,000 \mathrm{~km}$; and the SSV, which extends from $3,000 \mathrm{~km}$ to $36,000 \mathrm{~km}$, or approximately geostationary altitude [2].

\footnotetext{
${ }^{1}$ Aerospace Engineer, Navigation and Mission Design Branch, NASA GSFC.

${ }^{2}$ President, FBauer Aerospace Consulting Services.

${ }^{3}$ Aerospace Engineer, Navigation and Mission Design Branch, NASA GSFC.

${ }^{4}$ Aerospace Engineer, Navigation and Mission Design Branch, NASA GSFC.
} 
Continuous availability of at least four GPS signals has become a standard expectation for space users within the TSV, the regime which includes much of low Earth orbit. Similar to terrestrial users, space users in the TSV enjoy uniform received power levels and have fully overlapping coverage from the main beams of the GPS satellites, providing full coverage with at least 4 signals in view and instantaneous navigation solutions.

As space users enter the SSV, a number of changes occur to signal strength and signal availability which challenge GPS navigation and time sensing. Fig. 1 provides an illustrative two-dimensional depiction of a user spacecraft in a highly eccentric High Earth Orbit (HEO) within the SSV. While in the MEO portion of the SSV, from 3,000-8,000 km, GPS signals become stronger, due to shorter path lengths. Signal availability and performance is comparable to TSV users. As spacecraft altitude increases beyond these altitudes - into the HEO/Geosynchronous Earth Orbit (GEO) portion of the SSV - the number of available GPS mainlobe signals, depicted in dark yellow, begins to decrease rapidly due to poor geometry and blockage of main beam reception by the Earth. This leads many SSV users to consider using the full aggregate signal, including both mainlobes and sidelobes. Sidelobe signals (depicted in light yellow) can extend out to angles of up to $120^{\circ}$, substantially improving signal geometry and signal availability. By employing the aggregate signal, near-continuous availability of four or more simultaneous signals is realized. Moreover, the improved geometry from the wider sidelobe signals also improves navigation accuracy due to enhanced dilution of precision (DOP). Another challenge is GPS signal strength variations. As the user spacecraft traverses through this orbit, signal strength varies widely, becoming stronger as the user approaches GPS constellation altitudes, around 20,000 km, and then becoming significantly weaker above the GPS constellation altitude, where users must rely on signals from GPS satellites on the other side of the Earth. To overcome these challenges, special GPS receivers have been developed employing algorithms that enable acquisition and tracking of weak signals and generation of PNT solutions from less than four GPS signals in view.

To ensure best performance for navigation and timing applications at HEO, GEO, and beyond, mission designers strive for a minimum of one GPS satellite in view at all times. Modern onboard orbit estimation and propagation software, such as NASA's Goddard Enhanced Onboard Navigation System (GEONS), can process individual measurements when less than four GPS signals are available, and propagate or "flywheel" through signal outages. When GPS signals are completely absent, however, propagated solutions will slowly degrade - or rapidly, if perturbations are present, such as after imperfectly executed or sensed maneuvers. Ensuring continuous availability of at least one signal for spacecraft in the SSV is a key performance parameter in providing robust PNT sensing for space vehicles.

\section{B. Emerging SSV User Types and Benefits from Real-time GPS Navigation in the SSV}

GPS navigation and time sensing in the SSV is a game-changer, enabling unprecedented new space mission types, and significantly improving the capabilities and performance of current mission types that elect to include GPS in their mission portfolio. Fig. 2 provides a summary of space mission applications that are enabled by precision GPS navigation signals in the SSV. These include Earth remote sensing missions in GEO requiring precise geolocation, space weather satellite constellations, launch vehicles and spacecraft traveling into cislunar space, formation flying and proximity operations missions, and others. Performance enhancements from recent operational missions such as the Geostationary Operational Environmental Satellite R (GOES-R) series Earth weather satellites and the Magnetospheric Multiscale Mission (MMS) space weather constellation are described in the next section. A survey of civil applications of GPS in the SSV is covered in a follow-on section. It is important to note that the current operational missions, MMS and GOES, would not have been possible without the efforts of the GPS program to 
protect signal availability in the SSV, through formal SSV definition and mainlobe specification. Such efforts to guarantee minimum GPS signal availability in the SSV will ensure widespread use of GPS - in the SSV and at altitudes beyond.

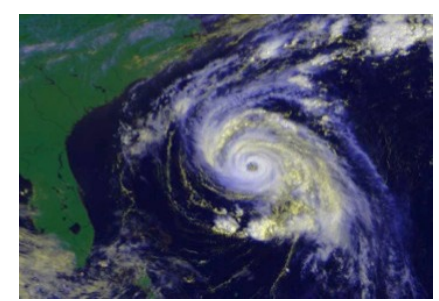

Earth Weather Prediction using Advanced Weather Satellites

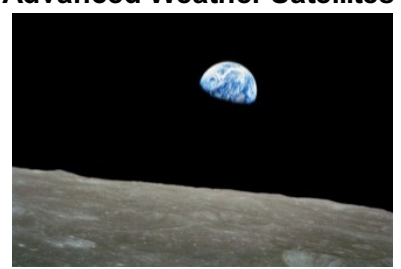

Launch Vehicle Upper Stages \& Beyond-GEO applications

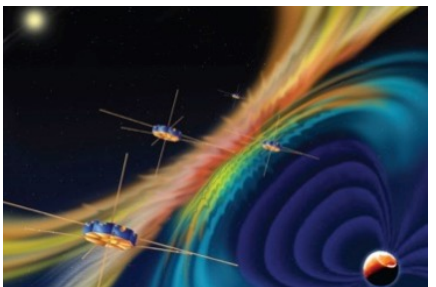

Space Weather Observations

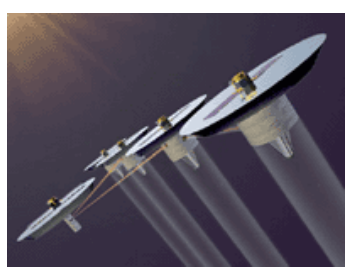

Formation Flying, Space Situational Awareness, Proximity Operations

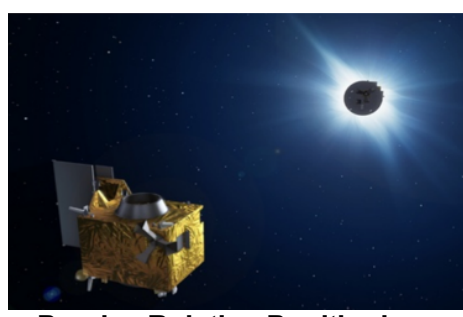

Precise Relative Positioning

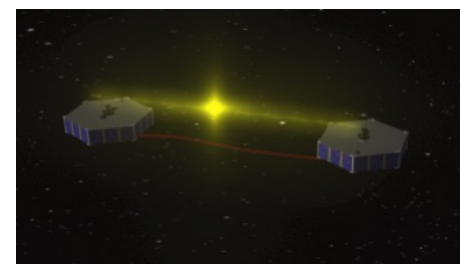

Precise Position Knowledge \& Control at GEO

Fig. 2 SSV space mission applications.

When compared to standard ground-based navigation, missions employing GPS in the SSV derive the following benefits:

\section{Fast recovery from trajectory maneuvers}

Improvement: From 5-10 hours to minutes.

2. Improved operations cadence

Improvement: From standard ranging ops cadence (e.g., daily updates for GOES) to real-time cadence with reduced/no tracking, quicker response to anomalies, fewer shifts, less specialized training, lower software license costs.

3. Increased satellite autonomy

Improvement: Enables formation flying. Reduction or elimination of ground station tracking and ground-based orbit determination lowers mission costs, estimated to be on the order of $\$ 500 \mathrm{~K}-750 \mathrm{~K}$ per year for multispacecraft formation flying missions.

4. Improved navigation performance including position, velocity, and navigation stability (or navigation jitter)

Improvement: Performance is mission and retrieval rate dependent. Examples include improvements from kmclass to 1-10 meter-class positioning; navigation stability improvements from not achievable, to 3-70 meters for rolling stability segments of 30 seconds to 30 minutes.

5. Precise timing reducing the need for expensive onboard clocks

Improvement: Savings is mission dependent. For precise timing requirements, savings from $\$ 50 \mathrm{~K}$ for Oven Controlled Crystal Oscillators or $\$ 15 \mathrm{~K}$ for Voltage Controlled Crystal Oscillators to hundreds of thousands to a million dollars for more precise solutions.

\section{Recent Experiences}

Numerous missions have been flown in the SSV with the ability to track GPS signals. The earliest of these dates back to 1997, when the first experiments were performed in a geosynchronous transfer orbit, followed by AMSAT OSCAR-40 in 2000 and GIOVE-A in 2005 [3]. More recently, there has been a transition from experimentation to operational utilization with reported usage by the United States [4], European Union (EU) [5], and Russia [6]. Here we explore recent published experiences by two civil operational users: MMS and GOES-16. 


\section{A. MMS - GPS Navigation at $\mathbf{4 0 \%}$ Lunar Distance}

NASA's MMS mission is a Solar Terrestrial Probe tasked with studying the phenomenon of magnetic reconnection in both the Earth's day-side magnetopause and night-side magnetotail. To obtain in-situ measurements of reconnection events, MMS employs four identical spacecraft, each with a suite of instruments, flying in a tetrahedral formation at apogee with scale distances ranging from $7 \mathrm{~km}$ to $160 \mathrm{~km}$ between spacecraft, and each spinning at $3 \mathrm{RPM}$. Launched on March 12, 2015, MMS entered a 1.2 $\mathrm{R}_{\mathrm{E}} \mathrm{x} 12 \mathrm{R}_{\mathrm{E}}$ (Earth Radii) "Phase 1" orbit for the magnetopause campaign, then in 2017 transitioned through an apogee-raising "Phase 2A" period to achieve a "Phase 2B" orbit of $1.2 R_{E} \times 25$ $\mathrm{R}_{\mathrm{E}}$ for the magnetotail campaign. A schematic of the two science orbits is shown in

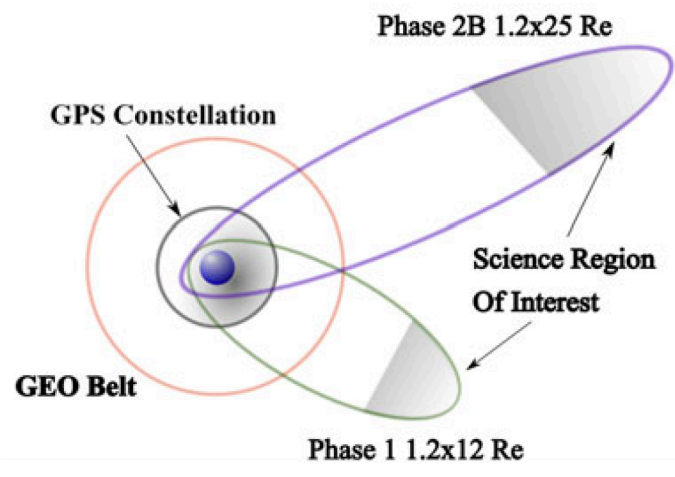

a) MMS primary mission science orbits

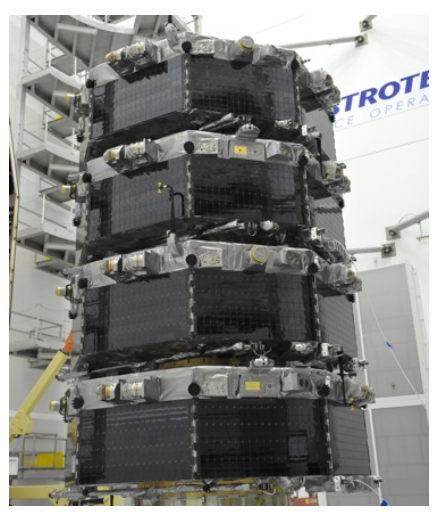

b) MMS 4-spacecraft stack prior to $L V$ integration

Fig. 3a [7].

Fig. 3 MMS spacecraft and primary mission science orbits.

The MMS design includes stringent navigation requirements, primarily on the ability of the system to estimate semi-major axis better than $50 \mathrm{~m}$ above $3 \mathrm{R}_{\mathrm{E}}$ in Phase 1 and better than $100 \mathrm{~m}$ in Phase 2B. Absolute position knowledge was required to be within $100 \mathrm{~km}$ root sum of squares (RSS). Early navigation development studies concluded that ground tracking alone could not meet the necessary formation flying requirements, leading the mission to select an onboard autonomous navigation solution consisting of the NASA Goddard Space Flight Center (GSFC) Navigator weak-signal GPS receiver, which features a $25 \mathrm{~dB}-\mathrm{Hz}$ tracking threshold, GEONS onboard Extended Kalman Filter (EKF), and (briefly) a crosslink ranging and alarming system, which was later dropped to reduce mission risk and complexity. This left onboard filtered GPS as the only navigation solution for the mission. As highaltitude performance of the GPS system, especially of the sidelobe signals, was not well known at the time, pre-flight simulations were conducted with highly conservative assumptions, and additional hardware was added to ensure adequate performance. The final system per-spacecraft consisted of two (primary and redundant) Navigator GPS receivers with GEONS embedded, and each with an ultra-stable crystal oscillator by Frequency Electonics, Inc., four custom GPS antennas with 4dBi peak gain, and four front-end electronics assemblies by Delta-Microwave, Inc. [7].

The first on-orbit results from the MMS navigation system were obtained during system checkout in the first three months of the mission during a time period spanning days 73-137 of 2015 (March 14-May 17). Fig. 4a shows the GPS signal visibility achieved over the first five days of the mission, including four full orbits. Visibility observed by all four spacecraft is plotted, but tracking performance was nearly identical so the four traces mostly overlap. At perigee, the receiver was tracking on all 12 channels, as expected. But at apogee, at $12 \mathrm{RE}$, or approximately twice the altitude of the geostationary belt, the average number of signals tracked simultaneously exceeded 8 signals, with no instances in which less than 4 were tracked simultaneously. This result was well beyond preflight predictions based on conservative assumptions and limited knowledge of the actual transmit power and transmit antenna gain patterns of the GPS constellation. Geometric DOP (not shown) exceeded 100 at times near apogee, so even though point solutions were obtained throughout Phase 1 , the filtered solution was used for navigation. Fig. $4 \mathrm{~b}$ shows the position and velocity RSS root-covariance (formal errors) obtained during the same period. After the initial convergence 
period, the peak position formal error is roughly $10 \mathrm{~m}$ near apogee, and the velocity formal error remains less than approximately $1 \mathrm{~mm} / \mathrm{s}$ except near perigee, where it spikes momentarily to $3 \mathrm{~mm} / \mathrm{s}$. These results were taken to bound the actual errors based on preflight testing and the results of an on-orbit navigation certification campaign using independent measurements [7].

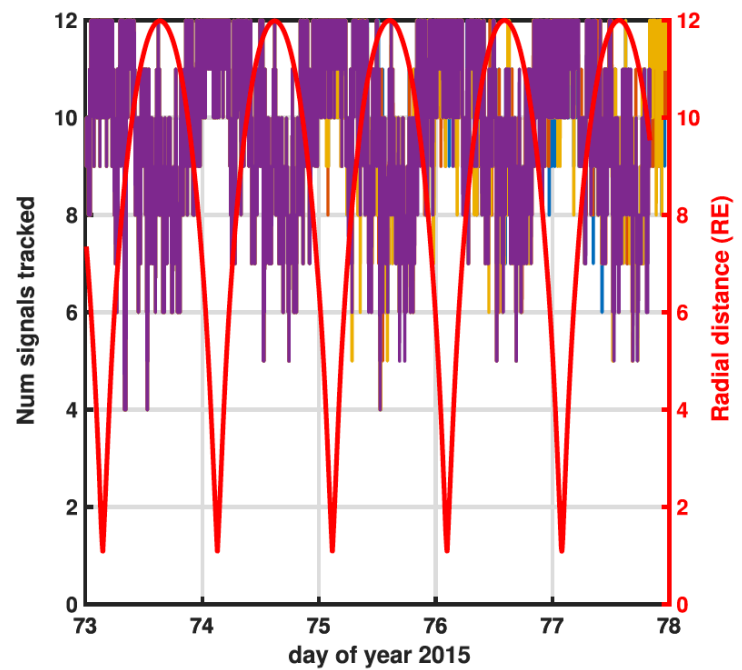

a) Phase 1 GPS signal visibility and orbit radial distance
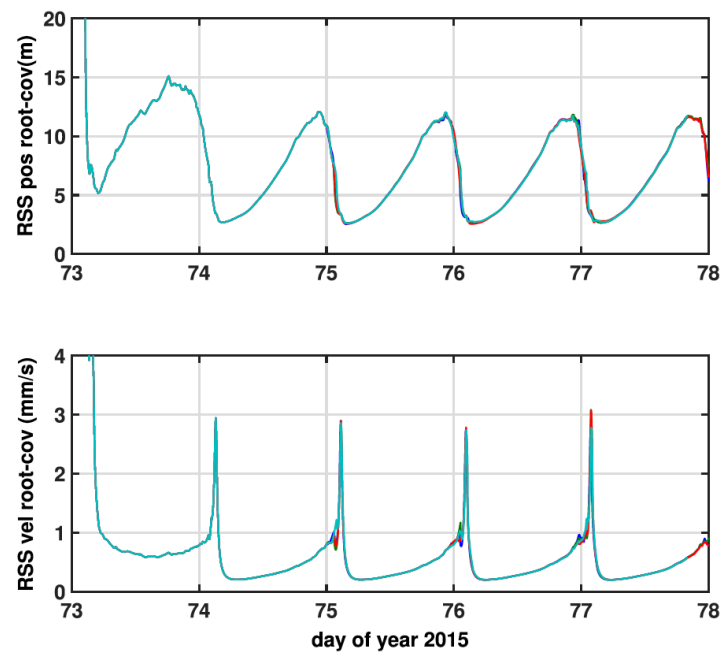

b) Phase 1 RSS position/velocity $1 \sigma$ formal errors

Fig. 4 MMS Phase 1 (12 $R_{E}$ apogee) GPS visibility and navigation performance.

Fig. 5 shows the same results for a representative period across three orbits in Phase 2B, when the apogee was 25 $\mathrm{R}_{\mathrm{E}}$, more than $40 \%$ of average lunar distance. Fig. 5a shows signal visibility measured by a single MMS spacecraft overlaid with orbit radius obtained in Phase 2B. Here, all 12 channels are tracking around perigee, and an average of 3 signals are tracked simultaneously at apogee, with only sporadic outages of short duration. Fig. $5 \mathrm{~b}$ shows the RSS root-covariance (formal errors) for both position and velocity, which do not exceed approximately $55 \mathrm{~m}$ and $2.5 \mathrm{~mm} / \mathrm{s}$, respectively. Again, velocity formal errors generally remain under $1 \mathrm{~mm} / \mathrm{s}$, only spiking briefly at perigee [8].

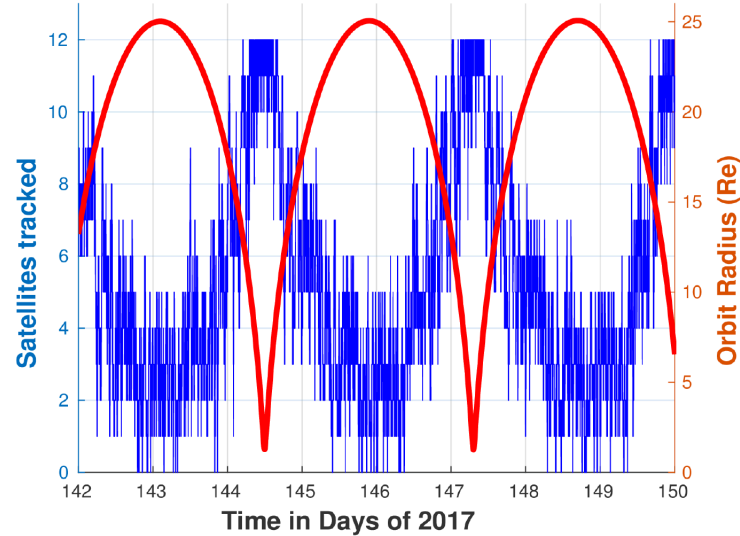

a) Phase $2 B$ GPS signal visibility and orbit radial distance

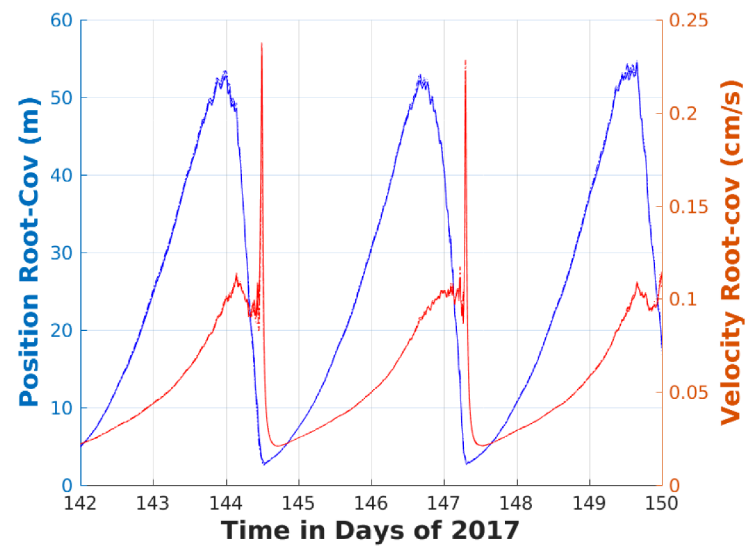

b) Phase 2B RSS position/velocity $1 \sigma$ formal errors

Fig. 5 MMS Phase 2B (25 RE apogee) GPS visibility and navigation performance.

To-date, MMS navigation performance has significantly exceeded both requirements, as shown in Table 1, and pre-flight predictions. The primary contributor to this exceptional performance has been the availability of the GPS sidelobe signals, which make up the large majority of signals tracked by MMS. While previously known to exist, they were of unknown strength and consistency across GPS blocks, and were of unknown navigation quality. MMS has proven that these signals are highly available (even to its highest altitude) and are of sufficient quality to contribute to high-quality navigation solutions, even when they make up the majority of the measurements. Direct simulations by the MMS team have shown that without sidelobe availability, position formal errors during 
Phase 1 would likely have doubled without maneuvers and grown even greater with maneuvers included. The MMS team is currently evaluating concepts for extended missions, one of which would have the apogee raised to $60+R_{E}$, or roughly lunar distance. After calibration with Phase 2B results, simulations indicate that GPS signals will be available even at this altitude without any change to the receiver, and that position formal errors will rise to the 1-2 km level (dominated by radial error, which becomes highly correlated with clock errors) [8].

Table 1. MMS Phase $1 \&$ Phase 2B navigation performance vs. requirements

\begin{tabular}{l|lcc}
\hline Description & Requirement & $\begin{array}{l}\text { Phase 1 } \\
\text { Performance }\end{array}$ & $\begin{array}{l}\text { Phase 2B } \\
\text { Performance }\end{array}$ \\
\hline Semi-major axis estimation under 3 $\mathrm{R}_{\mathrm{E}}(99 \%)$ & $50 \mathrm{~m}$ (Phase 1), & $6 \mathrm{~m}$ & - \\
& $100 \mathrm{~m}$ (Phase 2B) & - & $15 \mathrm{~m}$ \\
\hline Orbit position estimation (99\%) & $100 \mathrm{~km}$ RSS & $65 \mathrm{~m}$ & $55 \mathrm{~m}$ \\
\hline
\end{tabular}

\section{B. GOES-16-GPS at GEO Enabling Next-Generation Weather Observation}

GOES-16 is the on-orbit designation for the first of the GOES-R series of NASA/NOAA GEO weather satellites (see Fig. 6), which will consist of four spacecraft launched between 2016 (GOES-R/GOES-16) and 2024 (GOES-U). The series is the fourth generation of the GOES program and represents a major leap forward in capability and technology. Its primary Earth-observing instrument, the Advanced Baseline Imager, provides three times the number of spectral bands, four times the resolution, five times the observation rate, and 100 times the data rate as the previous GOES-N series. As a result, it promises to enable accurate weather prediction through the National Weather Service of 5-7 days, a full two days greater than the current capability, along with numerous other specific improvements and new capabilities [9].

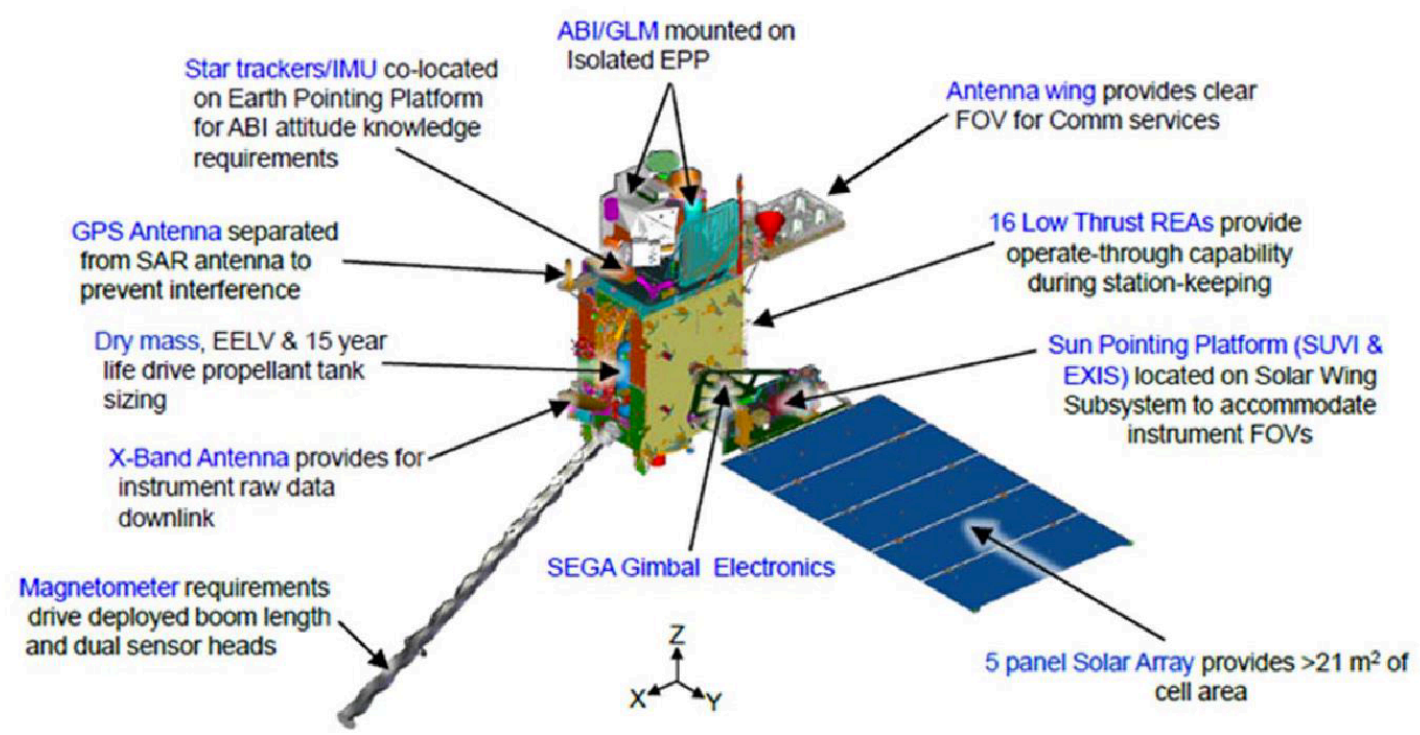

Fig. 6 GOES-R series deployed configuration. Reprinted from "Performance Characterization of GOESR On-Orbit GPS Based Navigation Solution" by M. Concha, et al., 2017, AAS Guidance and Control Conference 2017. Source: Lockheed Martin Space Systems Co. (LMSSC). Reprinted with permission.

The image navigation and registration (INR) requirements for GOES-R series are stringent, including orbit position and velocity knowledge of 75-100 $\mathrm{m}(3 \sigma)$ and $6 \mathrm{~cm} / \mathrm{s}(3 \sigma)$, respectively, limits on navigation "jitter" over specific intervals, and continuity through daily station-keeping and momentum management maneuvers with less than 120 min of lost observation time per year [9],[10]. Early studies identified that navigation via ground-based ranging would be impractical; instead, an onboard autonomous navigation system based on GPS was chosen. The resulting system consists of a specially-designed General Dynamics Viceroy-4 high-altitude GPS receiver and Low Noise Amplifier, coupled with a GPS receive antenna designed by Lockheed Martin specifically for use at GEO. The antenna gain pattern is shown in Fig. 7; its primary features include a peak gain of approximately $11 \mathrm{dBi}$ centered at $22^{\circ}$ offboresight. When mapped to the GPS transmit antenna gain patterns, the peak receive gain is aligned with the GPS first sidelobe, maximizing coverage of the sidelobe regions. The receiver itself features an embedded navigation filter and 
a minimum carrier-to-noise-density ratio $(\mathrm{C} / \mathrm{N} 0)$ of $17 \mathrm{~dB}-\mathrm{Hz}$, enabling sparse signal utilization and reception of weak sidelobe signals [11].

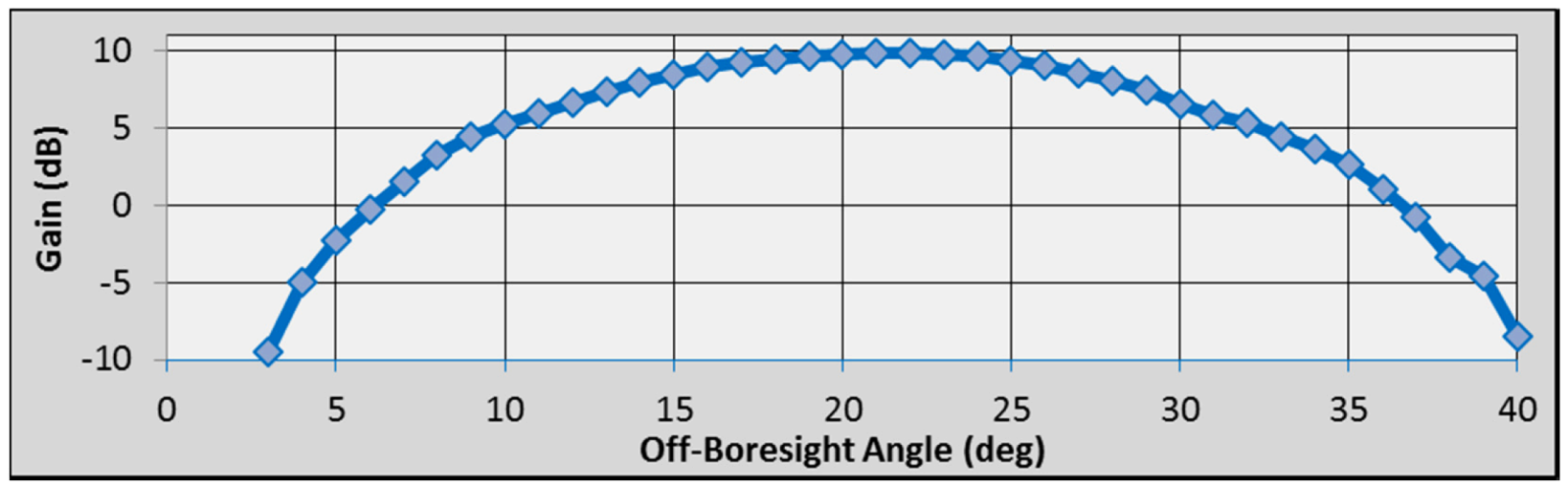

Fig. 7 Gain pattern of GOES-R series GPS receive antenna. Reprinted from "GPS Receiver On-Orbit Performance for the GOES-R Spacecraft" by S. Winkler, et al., 2017, 10th International ESA Conference on Guidance, Navigation \& Control Systems, Salzburg, Austria. Reprinted with permission.

The primary public source for in-flight GOES-16 performance data is a 50-hour span recorded on 2-4 Feb 2017, during the Post Launch Testing campaign. During the period, the spacecraft was controlled within $\left(0.007^{\circ}\right.$ lat., $0.035^{\circ}$ lon., $12 \mathrm{~km}$ alt.) centered at its $\left(0^{\circ}\right.$ lat., $-89.484^{\circ}$ lon., $35786 \mathrm{~km}$ alt.) station via two North-South stationkeeping maneuvers occurring about 24 hours apart, and each lasting approximately 11 minutes with a magnitude of $2 \mathrm{~cm} / \mathrm{s}$. Fig. 8 shows both the number of signals tracked (bottom plot), and the DOP (top plot) over the span. The average number of signals tracked is greater than 11, with all channels of the 12-channel receiver tracking for much of the time. Seven signals or more are tracked over the entire span. This is exceptional performance in the context of conservative pre-flight estimates and the formal specifications for signal visibility at GEO but is consistent with results seen by MMS. The DOP over the span ranges from 5 to 15 , only slightly above the theoretical minimum of 4 for a receiver in GEO, and also much better than expected. Both results increase the likelihood that the on-orbit navigation solution (whether via receiver point solutions or a filtered solution) will be of high quality [11].

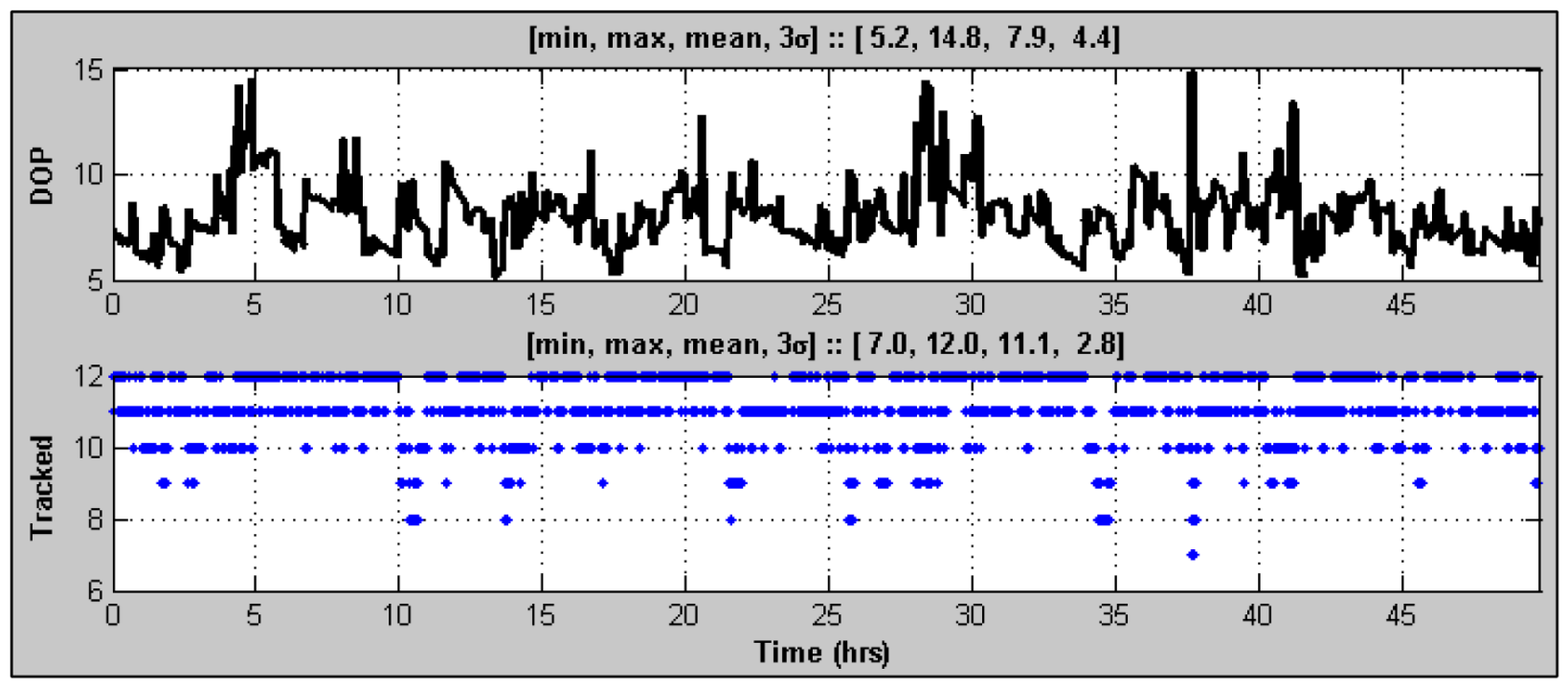

Fig. 8 GOES-16 on-orbit GPS visibility and DOP. Reprinted from "GPS Receiver On-Orbit Performance for the GOES-R Spacecraft” by S. Winkler, et al., 2017, 10 $^{\text {th }}$ International ESA Conference on Guidance, Navigation \& Control Systems, Salzburg, Austria. Reprinted with permission.

The on-orbit navigation performance was evaluated by comparing it to both a calibrated hardware-in-the-loop simulation, and to a ground filtered solution based on downlinked GPS receiver telemetry. Fig. 9 shows the result 
of the latter method, which is considered the most accurate. Using thrust data for the two maneuvers, modeling biases, and using highly accurate post-processed GPS ephemerides, a smoothed "truth" ephemeris was generated by the ground-based EKF and differenced with the onboard ephemeris generated by the receiver. The ground EKF solution has a variance of $3 \mathrm{~m}$ (shown as dashed lines in Fig. 9) which can be included in the difference to maximize conservatism. The effect, however, is minor [11].

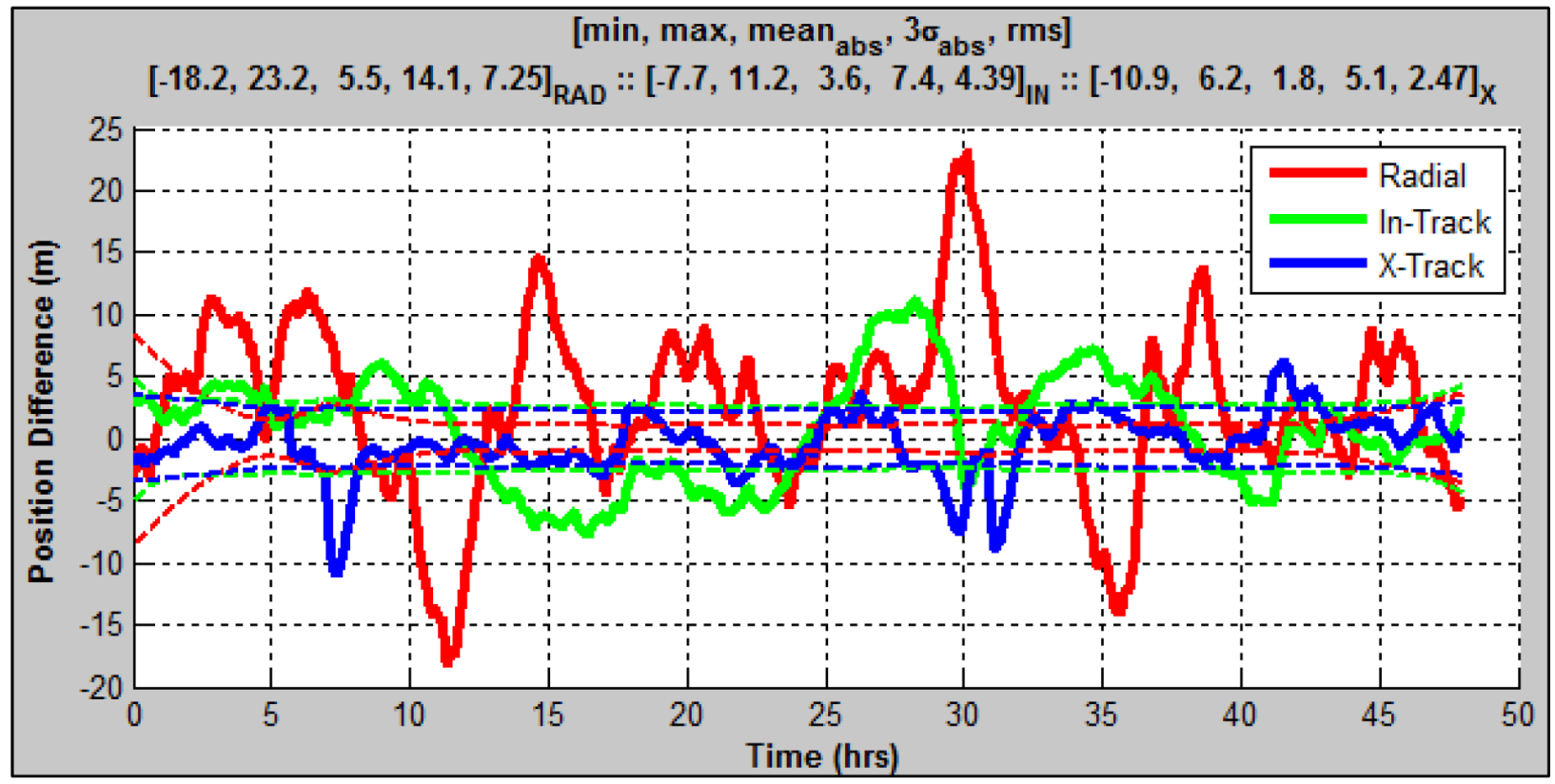

Fig. 9 Difference between on-orbit navigation solution and ground EKF solution (with ground 3 m variance shown in dashed lines). Reprinted from "GPS Receiver On-Orbit Performance for the GOES-R Spacecraft" by S. Winkler, et al., 2017, $10^{\text {th }}$ International ESA Conference on Guidance, Navigation \& Control Systems, Salzburg, Austria. Reprinted with permission.

Like MMS, GOES-16 is significantly exceeding its navigation requirements, as shown in Table 2, and pre-flight predictions. Also, like MMS, this performance is primarily thanks to the ability of the receiver to track the GPS sidelobe signals, which appear to make up approximately $80 \%$ of the signals tracked. This ability is a result of the combination of weak-signal tracking features of the receiver that allow it to track signals with $\mathrm{C} / \mathrm{N} 0$ values as low as $17 \mathrm{~dB}-\mathrm{Hz}$, and of higher-than-expected C/N0 values of the signals themselves, which were measured at $3 \mathrm{~dB}$ higher than expected from specifications. Prior NASA studies have shown that without the GPS sidelobe signals, the GPSbased orbit determination system on GOES-16 could not meet its navigation requirements [12]. The on-orbit performance of GOES-S through GOES-U is expected to be similar to that seen on GOES-16, barring any changes to the signals being broadcast by the GPS constellation itself. It is due to this performance that the GOES-16 team recommends that all future GEO satellites consider use of GPS for navigation to take advantage of available capability [11].

Table 2 GOES-16 key navigation performance vs. requirements

\begin{tabular}{|c|c|c|}
\hline Description & 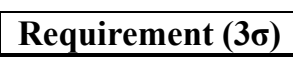 & Performance $(3 \sigma)$ \\
\hline Position knowledge, radial & $100 \mathrm{~m}$ & $20 \mathrm{~m}$ \\
\hline Position knowledge, in-track & $75 \mathrm{~m}$ & $13 \mathrm{~m}$ \\
\hline Position knowledge, cross-track & $75 \mathrm{~m}$ & $7.3 \mathrm{~m}$ \\
\hline Velocity knowledge & $6 \mathrm{~cm} / \mathrm{s}$ each axis & $0.69 \mathrm{~cm} / \mathrm{s} \mathrm{max}$ (cross-track) \\
\hline
\end{tabular}

\section{SSV Future Civil Applications}

A wide variety of future space applications stand to benefit from precision GPS navigation in high Earth orbits. In addition to the operational missions described in the previous section, numerous science missions employing GPS 
in the SSV are being proposed or are in development. These include Earth remote sensing missions in GEO requiring precise geolocation, space weather satellite constellations, launch vehicles and spacecraft traveling away from Earth, formation flying and proximity operations missions, and others. Specific mission types, their mission objectives, and their GPS SSV needs are described below.

\section{A. Earth Weather Missions}

The GOES-R weather satellite series in the United States (Fig. 10), and similar Earth weather satellite series in Europe (Meteosat Third Generation) [13] and Russia (Elektro-L) [6], are key examples of national-level GEO remote-sensing spacecraft that currently utilize (GOES-16, Elektro-L) or are studying (Meteosat) GPS for precise geolocation. These new, GPS-enabled Earth weather spacecraft are providing transformative societal benefits by protecting people and property through improved weather prediction and operational early warnings of a significant number of diverse natural hazards, including tornados, flash floods, wildfires, etc. Moreover, scientists expect that this generation of spacecraft will enable reliable extended forecasting to stretch from 3-5 days now to 5-7 days, a change that will beneficially impact daily life around the world [14].

In general, Earth weather satellite missions develop data products that support weather prediction and modeling. This may also include supporting advanced warning civil defense messaging of natural catastrophic events (flash floods, tornados, etc.) that are spread through local emergency broadcast messages and sirens/alarms. The accurate geolocation that is afforded through GPS enables Earth weather missions to determine the exact location of downpours in mountainous areas, which supports accurate and timely flash flood warnings, and precise location of remote wild fires which enables the safe placement of firefighters and equipment on the right side of the fire outbreak. In the case of GOES-R series spacecraft, the primary innovation is the ability for the National Weather Service to more accurately measure composite wind velocity vectors by taking derivatives of cloud locations between multiple images to derive wind velocities and atmospheric convergence, divergence, and rotation. This can only be accomplished through the coupled effects of two innovations: higher instrument temporal cadences and resolution, and the continuous, precise navigation and increased navigation stability enabled by onboard GPS-based navigation.

In addition to the GOES-16 case described in detail in the previous section, Russia's Elektro-L spacecraft have been using combined GPS/GLONASS navigation since 2011, with the second spacecraft in the series launched in 2015. Similarly, in 2008 EUMETSAT published the results of a trade study for navigation of its next generation of national GEO weather satellites, Meteosat. The third generation, which will consist of four spacecraft with an initial launch in 2021, will feature increased resolution and temporal cadence over the previous generation, and also an orderof-magnitude more stringent geolocation requirement, from $3 \mathrm{~km}$ to $250 \mathrm{~m}$. Global Navigation Satellite System (GNSS) based navigation was studied to provide accurate navigation to meet these requirements, and was predicted to achieve $360 \mathrm{~m}(3 \sigma)$ position knowledge utilizing main-lobe signals only. A comparison of this result to the on-orbit experience of GOES-16 illustrates the enabling importance of also utilizing the sidelobe signals. It should be noted that as weather forecasters continue to drive towards even more accurate weather prediction, follow-on national weather satellite systems currently being conceived will drive towards even more stringent requirements than these.

\section{B. Space Weather and Heliospheric Science Missions}

Space Weather and Heliospheric Science missions investigate the science of the Sun-Earth connection in order to deepen our understanding and, ultimately, prediction of space weather. It includes the origin and evolution of the solar wind, low-energy cosmic rays, and the interaction of the Sun's heliosphere with the Earth's magnetic field and local interstellar medium. Understanding the dynamics of these systems in three dimensions, especially during solar storms, is critically important for space weather prediction. Space weather missions operate throughout cislunar space and beyond. Many are in highly elliptical, high Earth orbit or in geostationary orbit.

The four MMS formation flying spacecraft represent an operational example of a space weather science mission employing GPS in the SSV. Candidate future missions include follow-on missions to MMS (with additional formation flying autonomy and inter-satellite communications), the Solar Dynamics Observer (Sun Observer, GEO), Polar (magnetospheric/plasma science, HEO), and THEMIS (Earth magnetotail substorm investigation, HEO). A magnetospheric constellation is another candidate follow-on mission that would scatter sentinels throughout the 
magnetosphere. Additionally, many space weather CubeSats, some in constellations or as formation flyers, have been proposed. Many of these missions will include onboard GPS for PNT sensing and control.

Space weather storms have the potential to disable on-orbit spacecraft, on-orbit and ground-based electronics, wireless communications, the GPS ground and/or space segments, electrical grids, etc. They can also impact the health of astronauts in space through the exposure to ionizing radiation. The 1859 solar storm, the so-called "Carrington event," was so severe it impeded global telegraph operations. Space Weather missions are enabling a better understanding and, ultimately, prediction of solar storms. It is important to be able to predict space weather events in order to reduce potentially harmful impacts to infrastructure and economic effects to society. Space weather observations are quickly transforming from single point observations to multi-point, three dimensional observations. Through this new vantage point, scientists are able to better understand how solar storms affect the dynamics of the magnetosphere and how these dynamics impact ground and on-orbit infrastructure, assets, and human health.

GPS navigation and timing capabilities have several benefits to space weather missions. Fast recovery from trajectory maneuvers and improved navigation performance afforded by GPS (e.g. 10-meter to 1-meter class) enable accurate determination of the location and motion of space weather phenomenon, including dynamic variations between formation flying spacecraft. Improved operations cadence and increased satellite autonomy reduces satellite operations costs, enhances science observations, and reduces mission risk during anomalies via quicker recovery. And with a precise time base from GPS, many space weather missions will be able to forgo expensive USOs for event timing and employ GPS as a less expensive alternative.

\section{Satellite Servicing}

Robotic satellite servicing can extend the life of a spacecraft through upgrade, repair, refueling, and orbit adjustment. It can also be used for debris removal and in-orbit construction or installation. Commercial and government entities are considering a number of targets, both civilian and military, with a particular focus on the relatively dense geostationary regime. The United States' Defense Advanced Research Projects Agency (DARPA) has been involved in satellite servicing research for over a decade [15], and its Robotic Servicing Geosynchronous Satellites program intends to demonstrate servicing technologies on orbit in 2021 [16] [17]. NASA's Satellite Servicing Projects Division is developing a LEO technology demonstration mission, Restore-L, that will service Landsat 7 in 2020 [18]. Space Logistics LLC's Mission Extension Vehicle ${ }^{\mathrm{TM}}$ is scheduled to service the Intelsat-901 spacecraft early next year [19] [20], UK-based Effective Space plans to launch a GEO servicing mission in 2020 [15], while the European Space Agency (ESA) and others are making significant investments in the field [21][22].

Regardless of the particular application, a typical concept of operations is as follows: a robotic servicer using GPS for PNT, as well as other sensors for relative pose estimation, performs autonomous rendezvous and docking with the target. The target may be cooperative or uncooperative and may have challenging attitude and angular momentum initial conditions. The servicer then repairs or refuels the target spacecraft and/or modifies its orbit.

Satellite servicing has the potential to significantly reduce mission lifecycle costs or the cost of additional satellite replacement and launch, but such activities place stringent demands on the GPS SSV. Fast recovery from trajectory maneuvers is required - on the order of minutes during critical rendezvous, proximity operations and docking. Near continuous GPS signal availability is needed to support satellite responsiveness and autonomy. And finally, highly accurate absolute orbit state (position and velocity) are necessary to support far-field rendezvous. As a general rule of thumb, position must be known to an accuracy of $10 \%$ the inter-vehicle range [23]. Although other sensors, such as camera and LIDAR, may be used during the final stages of docking in order to meet this requirement, GPS is critical during rendezvous and initial approach. Restore-L, for instance, requires GPS-only absolute accuracy of $30 \mathrm{~m}$ in position and $30 \mathrm{~cm} / \mathrm{s}$ velocity 3 -sigma, regardless of separation distance.

\section{Formation Flying Missions}

This mission class supports systems that will be using extensive autonomous navigation and trajectory control systems to support formation flying, cluster flight, and autonomous constellation control. Missions in this class span many different vehicle sizes, from CubeSats to missions flying in formation or docking with the International Space Station or the future Deep Space Gateway. They also span a myriad of different objectives in MEO, HEO or GEO, including heliospheric formation flying missions, such as MMS, robotic servicing and debris collection, GEO Earth science formation flyers, GEO hosted payload formations, solar chronographs, and formation flyers performing gravity wave, exoplanet [24], dark energy, and x-ray science.

NASA, DARPA, Air Force Research Laboratory, and ESA have invested heavily in formation flying missions and technology, including system F6 (DARPA)[25], EO-1 (NASA)[26] [27], MMS (NASA), Prisma (ESA) [28] and Proba-3 (ESA) [29]. Formation flying will open new mission, science, and commercial opportunities through innovative, distributed data gathering techniques and unique ways to perform science observations. 
The Project for On-Board Autonomy-3 (PROBA-3) mission (Fig. 11) represents an outstanding upcoming ESA formation flying mission that plans to employ GPS in the SSV. PROBA-3 is a high altitude solar occultation mission using precise formation flying in a $600 \mathrm{~km}$ by $60,000 \mathrm{~km}$ orbit to perform detailed observations of the Sun's corona. The primary objectives of Solar occultation missions, or solar chronographs, are to enable scientists to perform detailed measurements of the Sun's corona. The Sun's corona is currently not well observed due to the fact that it is about a million times dimmer that the Sun. It can only be observed if an object the size of the Sun's disk is placed in front of the sun to occult the significantly brighter Sun's surface. In the past, this could only be accomplished during total solar eclipses. On PROBA-3, this will be accomplished using GPS and other sensors to fly two spacecraft in a precise, choreographed formation, with one spacecraft, a coronagraph, taking measurements and a second, a spherical shield, which serves to occult the Sun. Internationally, this solar science has great interest and could not be done without the use of GPS in the SSV.

As this is such a wide class of mission types, the GPS SSV PNT requirements span the entire spectrum of GPS needs. It requires near continuous GPS signal availability to support precise navigation and timing, fast recovery from trajectory maneuvers, enhanced operations cadence, and increased satellite autonomy. Requirements as low as meterclass navigation in real time, $\mathrm{cm}$-level relative navigation and micro- to nanosecond timing synchronization would support the above mission types.

\section{E. Commercial GEO Missions}

In space, the most coveted real estate is a GEO belt spot perched over the Earth's surface. Currently, the number of spacecraft in GEO is limited by the need to mitigate the risk of radio frequency interference and spacecraft collisions. From a navigation perspective, the primary limiting factors in tightening the GEO arc spacing is the spacecraft relative navigation errors from traditional ground-based navigation techniques, dispersion errors that occur after vehicle trajectory maneuvers, and the slow operations cadence required to perform ground orbit determination using traditional ground-based navigation techniques. With the use of autonomous, real-time PNT solutions afforded by GPS, these navigation-related issues are eliminated and closer satellite-to-satellite spacing becomes feasible.

Many missions in the GEO belt are station keeping with low thrust, high impulse ion thrusters. These continuously maneuvering missions would benefit significantly from the real-time orbit determination solutions enabled by GPS, as illustrated by the US, EU, and Russia moving to GPS/GNSS for national GEO weather missions. As the space community gains confidence and trust in the on-orbit control authority using GPS in the SSV, the density of spacecraft in the GEO arc will increase over time.

The ability to enhance the density of satellites in the GEO arc will have tremendous, positive benefits to commercial and civil space users. The commercial GEO space market is a critical space infrastructure and a huge revenue stream, particularly for telecommunications applications. Flying spacecraft in GEO has provided significant commercial revenue for users such as Direct TV, AT\&T and many others. Many nations have spacecraft in this arc supporting Earth weather, telecommunications, and space weather observations.

\section{F. Launch Vehicle Upper Stages \& Deep Space Missions, En Route, and Return}

Launch vehicle use of real-time GPS navigation can enable more accurate trajectory insertion techniques, resulting in more efficient propellant use and higher payload capacities. This mission class includes launch vehicle upper stages that perform direct ascent burns into HEO, GEO, cislunar orbits, or deep space trajectories. These can include commercial upper stages (e.g., ULA, SpaceX) employed by commercial, NASA or international organizations. This mission class also includes upper stages supporting NASA's human spaceflight exploration Space Launch System missions beyond low Earth orbit and missions to and on the Lunar Orbital Platform-Gateway. This includes the Interim Cryogenic Propulsion Stage and the Enhanced Upper Stage. Launch vehicle upper stages strive for high accuracy, high cadence position, velocity, and time knowledge to minimize the trajectory propagation errors of the vehicle during flight. For launch vehicles with secondary payloads, utilization of GPS in the SSV can feed into secondary payload ejection schedules to increase the probability of achieving the desired orbit, as well as to aid in initial state estimation of the ejected payloads. 
NASA deep space missions can also benefit from GPS in the SSV to improve navigation accuracy. This leads to more accurate trajectory correction maneuvers, resulting in less required propellant and higher payload mass capability. This mission class can include a return capsule, such as the Mars Sample Return mission, Stardust or Genesis mission follow-ons, or a similar mission en route or returning from Lunar orbit, like the Lunar Cubequest challenge. These vehicles could employ GPS in MEO, HEO, GEO, and cislunar space. For those going into deep space, they would perform spacecraft navigation with GPS while outbound and inbound, then transition to other onboard techniques such as OpNav as they leave the vicinity of the Earth. For those vehicles inbound, significant reentry risks are mitigated through the acquisition of highly accurate GPS navigation solutions in the SSV. These solutions provide early and accurate navigation knowledge to precisely place the vehicle on the best reentry path.

Payload mass and accurate ejection are coveted capabilities of all launch vehicles. Early and accurate navigation is crucial for deep space missions as they lead directly into safe planetary transitions (e.g., reentry or orbit insertion) and additional payload or propellant capability. All these are enabled through the use of GPS in the SSV. Position navigation performance needs for these mission classes are on the order of 100-300 meters, well within the capability of GPS in the SSV.

\section{G. Lunar Missions}

There has been a renewed interest in lunar exploration, both as a target for rovers and landers, and as a first step toward deep space human exploration. Navigation for lunar-vicinity spacecraft has conventionally been performed on the ground, but the recent high-altitude missions discussed above have demonstrated operational, onboard, GPS-based navigation at GEO and beyond. It is clear that GPS could play an important role in the outbound and return phases of a lunar mission, providing measurements for mid-course correction burns at the upper limit of these altitudes [30] [31]. But simulations have shown that this can be extended to lunar distances by simply augmenting existing highaltitude GPS navigation systems with a high-gain antenna. A simulation of GPS signal availability on a lunar trajectory, calibrated with GOES-16 and MMS flight data, showed at least one signal was visible $99 \%$ of the time, with four or more visible out to $55 \mathrm{R}_{\mathrm{E}}$ [32]. The recalibrated MMS navigation system ground simulation demonstrated kilometer-level range errors, and $100 \mathrm{~m}$-level lateral errors $(3 \sigma)$ with existing receiver technology [8]. Other studies have shown promising signal availability at lunar distances [33] [34].

The United States plans to return to human exploration of the Moon and cislunar space in the next few years with the first two Exploration Missions, EM-1 and EM-2. These missions re-establish the fundamental capabilities necessary to take humans from the Earth, to cislunar space, and back again, first with an un-crewed distant retrograde orbit with EM-1, then with a crewed, free-return trajectory with EM-2. GPS could be an important navigation backup for the crew capsule, Orion, if the communications link is lost. EM-3 may begin construction of a "gateway" - a permanent way-station in the vicinity of the moon for staging deep space activity. All of the international partners involved in the International Space Station (ESA, NASA, Roscosmos, JAXA, and CSA) are engaged in early planning for a lunar gateway [35].

The gateway essentially extends the International Space Station (ISS) concept into the SSV, and the lessons learned with ISS in LEO should be incorporated. GPS would benefit a lunar platform like the gateway as both a part of the infrastructure and as a payload capability. As a source of position, velocity, and potentially attitude, GPS would enable periods of autonomy and provide the option of reducing reliance on Deep Space Network. Perhaps of equal importance, GPS would provide a stable and accurate timing source, an important resource for hosted science and technology payloads. This additional navigation and timing capability from GPS in the SSV can enable enhanced Rendezvous and Docking techniques that in turn enable in-space construction and assembly of assets outside of LEO, reducing delta-V needs to leave the Earth or Earth/Moon system.

\section{Next Generation SSV}

In order to ensure that the SSV capability is preserved and maximized for these and other uses, multiple efforts must be undertaken. Specific to GPS, the majority of the capability currently being utilized on-orbit exceeds that guaranteed by the constellation requirements, putting it at some risk of being modified by future designs of the GPS satellites. Block III satellites (SV1-10) will soon begin to populate the constellation and will affect the combined on-orbit performance. The performance of these satellites is expected to be similar to that of the previous blocks (especially Block IIR-M), so impacts are expected to be minor. However, the follow-on Block IIIF (SV11-32) has not yet been procured and is expected to be ready for launch in the mid-2020s [36]. The outcome of this procurement will dictate the capability of GPS in the SSV for decades to come.

Beyond GPS, expanding the concept of the SSV and the receiver capabilities of SSV users to include the other GNSS and Regional Navigation Satellite System (RNSS) constellations promises to significantly increase the 
available capability, both due to the larger number of signals available, and due to the variety of geometries inherent to these systems. The Russian GLONASS is largely complete but is undergoing modernization [37]. The European Galileo system will reach Full Operational Capability in 2019 [38], and the Chinese BeiDou Navigation Satellite System (BDS) will reach completion in 2020 [39]. BDS includes a geostationary component, increasing signal visibility to certain areas of the SSV. The two RNSS systems, Japan's QZSS and India's NAVIC, will augment SSV capability depending on geometry. Once combined, signal visibility at GEO due to only mainlobe signals will increase to $100 \%$ for any single signal, and will reach nearly $90 \%$ for four simultaneous signals, given a $20 \mathrm{dBHz}$ receiver tracking threshold and no applied receiver antenna gain. With sidelobe tracking included, signal reception may increase to dozens of signals simultaneously. The interoperability of GNSS constellations in the SSV is being coordinated via the United Nations International Committee on GNSS (ICG), which is working to publish formal performance expectations for each constellation in 2018, as a basis for establishing the capability of the multi-GNSS SSV.

The GPS and GNSS SSV concepts are both based on common definitions, including provision of signals via Earthpointed navigation satellites, typically via spillover of the mainlobe, and a general altitude ceiling at GEO for the purposes of specifying performance. In a future evolution of the SSV, both of these areas could be expanded dramatically. The signals themselves could be provided by numerous other methods, including:

- formalized and specified mainlobe and sidelobe signals from all constellations

- navigation via pseudorandom noise (PN) ranging on inter-satellite links (“cross-links")

- utilization of existing augmentation systems, such as WAAS and EGNOS

- SSV-specific augmentations, including satellites in supplementary locations or terrestrial or planetary beacons

With such augmentations, the user base could also be expanded beyond those discussed here to include new, highly-precise applications (benefiting from improved geometry), or extreme high-altitude applications, such as those beyond lunar orbit, to include Sun-Earth libration points, asteroid-belt objects, and beyond. The vision and future expansion of the SSV is a topic that is under active study by multiple organizations within the GNSS provider nations, and collectively by the ICG Working Group B, with the objective of establishing common, concrete goals that can be achieved multilaterally, for the benefit of all users.

\section{Conclusions}

Many emerging space missions are poised to benefit from precision navigation and timing afforded through the employment of GPS signals in the SSV and beyond - the volume of space starting at 3,000 km above the Earth and formally extending to geostationary altitudes. Currently, several operational missions use GPS in and above the SSV to improve their vehicle navigation performance, most notably the GOES weather satellites at GEO and the MMS four-spacecraft formation flying mission at altitudes up to $150,000 \mathrm{~km}$ or $40 \%$ of the way to the moon. The phenomenal results achieved from GPS on these missions have ushered the space community into the high-altitude GPS era.

Missions using GPS in the SSV take advantage of aggregate (main and sidelobe) signal reception to boost their navigation and timing performance. Use of sidelobe signals, in concert with mainlobe signals, improves overall signal availability and signal geometry for missions in the HEO and GEO regime. Through the employment of special weak signal GPS receivers and specially developed algorithms and software, spacecraft in high Earth orbit and within cislunar space now realize the following benefits: significantly reduced recovery time after trajectory maneuvers, improved operations cadence, increased satellite autonomy, and more precise, real-time navigation and timing performance. In total, these capabilities enable higher performing Earth and space science missions, reduced mission lifecycle costs (less hardware, reduced operations assets) and autonomous formation flying missions.

These benefits are illustrated by recent US civil missions like MMS and GOES-16, which both rely on onboard GPS navigation to meet (and exceed) stringent navigation requirements. MMS is currently demonstrating RSS

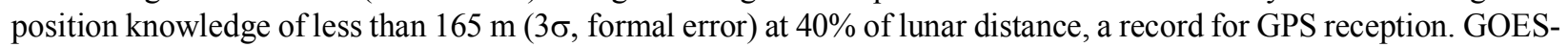
16 is the first of the fourth generation of US national GEO weather satellites and promises to deliver revolutionary improvements to national weather forecasting. It is demonstrating orbit position knowledge of $20 \mathrm{~m}(3 \sigma)$ or less, even through low-thrust daily station-keeping maneuvers, and with only 120 minutes of exceedance allowed per year.

Government and commercial mission applications enabled by precision GPS navigation in high Earth orbit include remote sensing in GEO requiring precise geolocation, Earth and space weather operational satellite constellations (e.g., GOES-16 \& MMS), space science missions, HEO/GEO spacecraft servicing missions, formation flying missions, and others. The lunar class and lunar gateway missions also expect to benefit from GPS. 
Humanity is poised to reap great benefits from the expansion and use of GPS in the SSV and beyond. This initiative will extend the routine use of GPS in space from low Earth orbits to HEO and GEO orbits and on into cislunar space. In its very short life-span, HEO GPS has already demonstrated outstanding operational and societal benefits. Its promise for the future is expected to be transformative, but only if the capability is fully realized. Users, service providers, and receiver developers must ensure that:

1. the critical capabilities of the existing SSV are protected and improved in the future,

2. international service providers cooperate fully on the expansion of the SSV to include contributions from all GNSS constellations,

3. technology developments support advanced and novel high-altitude receiver capabilities, including ultra-weak signal tracking and high-altitude onboard precise orbit determination, and

4. high-altitude users take advantage of the available capability to demonstrate application-specific benefits.

\section{Acknowledgments}

The authors would like to acknowledge the members of the NASA MMS team and the NASA, Lockheed Martin, and Relative Dynamics GOES-R teams for their support and review of the mission-specific Recent Experiences discussion included here, as well as the members of the NASA Restore-L team for their input. We would also like to recognize the countless people, military and civilian, that have been instrumental in the development and operation of the GPS system. Without their tireless efforts and attention to the details, employment of GPS in the SSV would not be possible.

\section{References}

[1] Bauer, F.H., Hartman, K.R., and Lightsey, E.G., "Spaceborne GPS Current Status and Future Visions," ION GPS-98, Nashville, TN, September 1998.

[2] Bauer, F.H., Moreau, M.C., Dahle-Melsaether, M.E., Petrofski, W.P., Stanton, B.J., Thomason, S., Harris, G.A, Sena, R.P., III, L. Parker Temple, "The GPS Space Service Volume," ION GNSS 2006, Fort Worth, TX, 2006.

[3] Verde, G., Unwin, M., Duncan, S., Hyslop, A., Kowaltschek, S., "Revisiting the SGR-GEO on GIOVE-A for GPS Satellite Antenna Pattern Mapping," GNC 2017: 10 th International ESA Conference on Guidance, Navigation \& Control Systems, Salzburg, Austria, May 2017.

[4] Miller, J. J., and Parker, J. J. K., "NASA GNSS Activities," Twelfth meeting of the International Committee on Global Navigation Satellite Systems, Working Group B, December 2017, Kyoto, Japan, URL: http://www.unoosa.org/documents/pdf/icg/2017/wgb/wgb_5.pdf.

[5] Bauer, F. H., Parker, J. J. K., Welch, B., Enderle, W., "Developing a Robust, Interoperable GNSS Space Service Volume (SSV) for the Global Space User Community," Proceedings of the 2017 International Technical Meeting of The Institute of Navigation, Monterey, CA, January 2017, pp. 132-149.

[6] Marareskul, D., "Space Service Volume and Russian GEO satellites PNT," United Nations International Committee on GNSS, Seventeenth Meeting of the Providers' Forum, Sochi, Russia, November $2016 . \quad$ URL: http://www.unoosa.org/pdf/icg/2016/icg11/pf17/pf1.pdf.

[7] Winternitz, L. B., Bamford, W. A., Price, S. R., Carpenter, J. R., Long, A. C., Farahmand, M., "Global Positioning System Navigation Above 76,000 KM for NASA's Magnetospheric Multiscale Mission", NAVIGATION, Journal of The Institute of Navigation, Vol. 64, No. 2, Summer 2017, pp. 289-300.

[8] Winternitz, Luke B., Bamford, William A., Price, Samuel R., "New High-Altitude GPS Navigation Results from the Magnetospheric Multiscale Spacecraft and Simulations at Lunar Distances," Proceedings of the 30th International Technical Meeting of The Satellite Division of the Institute of Navigation (ION GNSS+ 2017), Portland, Oregon, September 2017, pp. 1114-1126.

[9] Chapel, J., Stancliffe, D., Bevacqua, T., Winkler, S., Clapp, B., Rood, T., Freesland, D., Reth, A., Early, D., Walsh, T., Krimchansky, A., "In-Flight Guidance, Navigation, and Control Performance Results for the GOES-16 Spacecraft," GNC 2017: 10 ${ }^{\text {th }}$ International ESA Conference on Guidance, Navigation \& Control Systems, Salzburg, Austria, May 2017.

[10] Concha, M., Zanon, D., and Gillette, J., "Performance Characterization of GOES-R On-Orbit GPS Based Navigation Solution," AAS Guidance and Control Conference 2017, Feb 2017, Breckenridge, CO, AAS Paper 17-138.

[11] Winkler, A., Ramsey, G., Frey, C., Chapel, J., Chu, D., Freesland, D., Krimchansky, A., Concha, M., "GPS Receiver OnOrbit Performance for the GOES-R Spacecraft," GNC 2017: 10 ${ }^{\text {th }}$ International ESA Conference on Guidance, Navigation \& Control Systems, Salzburg, Austria, May 2017.

[12] Parker, J. J. K., Valdez, J. E., Bauer, F. H., Moreau, M. C., "Use and Protection of GPS Sidelobe Signals for Enhanced Navigation Performance in High Earth Orbit," AAS Paper 16-72, February 2016.

[13] Meixner, H., Righetti, P. L., Lagadec K., and Raballand, F., "Meteosat Third Generation Mission feasibility for Orbit and Attitude," SpaceOps 2008 Conference, May 2008, Heidelberg, Germany. doi: https://doi.org/10.2514/6.2008-3223 
[14] "The History and Evolution of the GOES-R Advanced Baseline Imager", Schmit, T.J.,GOES-R Program Mission Status Review Invited Presentation, March 26, 2015

[15] Henry, C., "DARPA satellite-servicing project comes under congressional fire," Space News, URL: http://spacenews.com/darpa-satellite-servicing-project-comes-under-congressional-fire/ [retrieved 26 March 2018].

[16] Roesler, G., "Robotic Servicing of Geosynchronous Satellites (RSGS)," Defense Advanced Research Projects Agency [website], URL: https://www.darpa.mil/program/robotic-servicing-of-geosynchronous-satellites [retrieved 26 March 2018].

[17] "Program Aims to Facilitate Robotic Servicing of Geosynchronous Satellites," Defense Advanced Research Projects Agency [website], URL: https://www.darpa.mil/news-events/2016-03-25 [retrieved 26 March 2018].

[18] "Restore -L Robotic Servicing Mission," SSPD [website], URL: https://sspd.gsfc.nasa.gov/ [retrieved 26 March 2018 ].

[19] Erwin, S., Henry, C., "Orbital ATK lands second Intelsat satellite servicing deal," Space News, URL: http://spacenews.com/orbital-atk-lands-second-intelsat-satellite-servicing-deal/ [retrieved $26 \mathrm{March} 2018$ ].

[20] "MEV-1: The Future of Satellite Servicing," Orbital ATK [website], URL: https://www.orbitalatk.com/news-room/featurestories/MEV_Progress/default.aspx [retrieved 26 March 2018].

[21] "Clean Space," ESA

[website], http://www.esa.int/Our_Activities/Space_Engineering_Technology/Clean_Space/e.Deorbit [retrieved 26 March 2018]

[22] "MDA Announces On-orbit Satellite Servicing Business Formation and Contract Awards for Spacecraft and First Life Extension Customer," SSL [website], URL: https:/www.sslmda.com/html/pressreleases/pr20170628.html [retrieved 26 March 2018].

[23] Fehse, Wigbert. Automated Rendezvous and Docking of Spacecraft. Cambridge University Press, 2008.

[24] Kolmas, J., et al., "System Design of a Miniaturized Distributed Occulter/Telescope for Direct Imaging of Star Vicinity" IEEE Aerospace Conference 2016.

[25] Hur-Diaz, S. and O'Connor, B., "Cluster Flight Application on System F6", International Symposium on Space Flight Dynamics, Johns Hopkins Applied Physics Laboratory, Laurel, MD, 2014.

[26] Bauer, F., Bristow, J., Folta, D., Hartman, K., Quinn, D., and How, J., "Satellite formation flying using an innovative autonomous control system (AutoCon) environment," in Proc. of the AIAA GNC, Aug 1997.

[27] Bauer, F., Hartman, K., Lightsey, E., "Spaceborne GPS: Current Status and Future Visions," ION GPS-98, Nashville, TN, September 1998.

[28] D'Amico, S., Ardaens, J.-S., and Larsson, R., "Spaceborne Autonomous Formation-Flying Experiment on the PRISMA Mission," Journal of Guidance, Control, and Dynamics, Vol. 35, No. 3 (2012), pp. 834-850.

[29] Llorente, J., et al., "PROBA-3: Precise formation flying demonstration mission," Acta Astronautica Volume 82, Issue 1, January 2013, Pages 38-46

[30] Barton, G., Sheppard, S., and Brand, T., "Proposed Autonomous Lunar Navigation System," Proceedings of AAS Guidance and Control Conference, Breckenridge, CO, February 1993.

[31] Lee, T., Long, A., Berry, K., Carpenter, J., Moreau, M., and Holt, G., "Navigating the Return Trip from the Moon Using Earth-Based Ground Tracking and GPS," Proceedings of AAS Guidance and Control Conference, Breckenridge, CO, February 2009.

[32] Ashman, B., Parker, J., Bauer, F., Esswein, M., "Exploring the Limits of High Altitude GPS for Lunar Missions," AAS GN\&C Conference, Breckenridge, CO, American Astronautical Society, February 2018.

[33] V. Capuano, C. Botteron, J. Leclare, J. Tian, Y. Wang, and P. Farine, "Feasibility Study of GNSS as Navigation Systems to Reach the Moon," Acta Astronautica, July 2015.

[34] E. Shehaj, V. Capuano, C. Botteron, P. Blunt, and P. Farine, "GPS Based Navigation Performance Analysis Within and Beyond the Space Service Volume for Different Transmitters? Antenna Patterns,” MDPI Aerospace, Vol. 4, August 2017, pp. $1-34$.

[35] "What is the Deep Space Gateway?" ESA [website], URL: http://exploration.esa.int/moon/59374-overview/ [retrieved 26 March 2018].

[36] Gleckel, G., "GPS Status \& Modernization Progress: Service, Satellites, Control Segment, and Military GPS User Equipment," Twentieth Meeting of the National Space-Based Positioning, Navigation, and Timing Advisory Board, November 2017. URL: https://www.gps.gov/governance/advisory/meetings/2017-11/gleckel.pdf

[37] ROSCOSMOS State Space Corporation, "GLONASS system development and use," Twelfth meeting of the International Committee on Global Navigation Satellite Systems, December 2017, Kyoto, Japan, URL: http://www.unoosa.org/documents/pdf/icg/2017/03_icg12.pdf.

[38] European Commission, "Galileo Programme Status Update," Twelfth meeting of the International Committee on Global Navigation Satellite Systems, December 2017, Kyoto, Japan, URL: http://www.unoosa.org/documents/pdf/icg/2017/04_icg12.pdf.

[39] China Satellite Navigation Office, "Update on BeiDou Navigation Satellite System," Twelfth meeting of the International Committee on Global Navigation Satellite Systems, December 2017, Kyoto, Japan, URL: http://www.unoosa.org/documents/pdf/icg/2017/05_icg12.pdf. 